multiple pipelines and use the results to obtain consensus findings. This could be achieved by implementing the type of meta-analysis used by Botvinik-Nezer and co-workers.

Another goal of the study was to evaluate how accurately researchers could predict the number of teams that would report significant results for each hypothesis. To study this, the authors ran separate 'prediction markets', one for the analysis teams and one for researchers who did not participate in the analysis. In them, researchers attempted to predict the outcomes of the scientific analyses and received monetary payouts on the basis of how well they predicted performance. Participants - even researchers who had direct knowledge of the data set - consistently overestimated the likelihood of significant findings. Botvinik-Nezer et al. do not explicitly explore how the analysts' prior beliefs affected their findings and pipeline choices. For example, if a research finding does not initially coincide with expectations, will groups seek to alter pipelines until expectations and results align? The prevalence of pipeline exploration implies that this is likely.

What other improvements could be made for the future? One approach is to use pipeline-optimization tools ${ }^{5,6}$ to reduce analysis flexibility. These tools automatically identify pipelines that maximize reproducibility, which can reduce the risk of excessive pipeline exploration and selective reporting. In addition, increased use of sensitivity analyses to evaluate the effects of pipeline decisions would provide a better understanding of the link between analysis choices and research findings. The fact that activity maps were highly correlated across groups implies that multivariate statistical approaches, which identify spatial patterns in the data, might provide more-consistent results across pipelines than would a series of tests performed at individual voxels.

Ultimately, neuroimaging results should be carefully verified using independent data sets to demonstrate generalizability across samples, research contexts and populations. A positive example of this already being done comes from an approach for developing predictive models on the basis of brain activation, which can be shared, tested in multiple contexts and used in applied settings ${ }^{7}$.

It seems unlikely that the fMRI field will ever coalesce on a standard workflow that is applicable to all types of study, because studies tend to be too varied for one pipeline to always be appropriate. But Botvinik-Nezer et al. conclude their paper by calling for an increased awareness of the situation, and a drive to improve the quality of method reporting. This is wise and prudent advice that researchers in any field analysing high-dimensional data would be well advised to heed.
Martin Lindquist is in the Department of Biostatistics, Johns Hopkins Bloomberg School of Public Health, Baltimore, Maryland 21205, USA.

e-mail:mlindqui@jhsph.edu
3. Carp, J. Front Neurosci. 6, 149 (2012)

4. Simmons, J. P., Nelson, L. D. \& Simonsohn, U. Psychol. Sci. 22, 1359-1366 (2011).

Strother, S. C. et al. Neurolmage 15, 747-771(2002).

6. Churchill, N. W. et al. Hum. Brain Mapp. 33, 609-627 (2012).

7. Woo, C. W., Chang, L. J., Lindquist, M. A. \& Wager, T. D. Nature Neurosci. 20, 365 (2017).
1. Carp, J. Neurolmage 63, 289-300 (2012).

2. Botvinik-Nezer, R. et al. Nature 582, 84-88 (2020).
This article was published online on 20 May 2020.

\title{
Optical physics
}

\section{Light turned into exotic Laughlin matter}

\section{Laura Corman}

A Laughlin state is a phase of matter that has remarkable features, such as excitations that behave as a fraction of a particle. The long-sought creation of a photonic Laughlin state is a milestone for the field of quantum simulation. See p.41

Solving a difficult physics problem can be surprisingly similar to assembling an interlocking mechanical puzzle. In both cases, the particles or pieces look alike, but can be arranged into a beautiful structure that relies on the precise position of each component (Fig.1). In 1983, the physicist Robert Laughlin made a puzzle-solving breakthrough by explaining the structure formed by interacting electrons in a device known as a Hall bar $^{1}$. Although the strange behaviour of these electrons still fascinates physicists, it is not possible to simulate such a system or accurately measure the particles' ultrashort

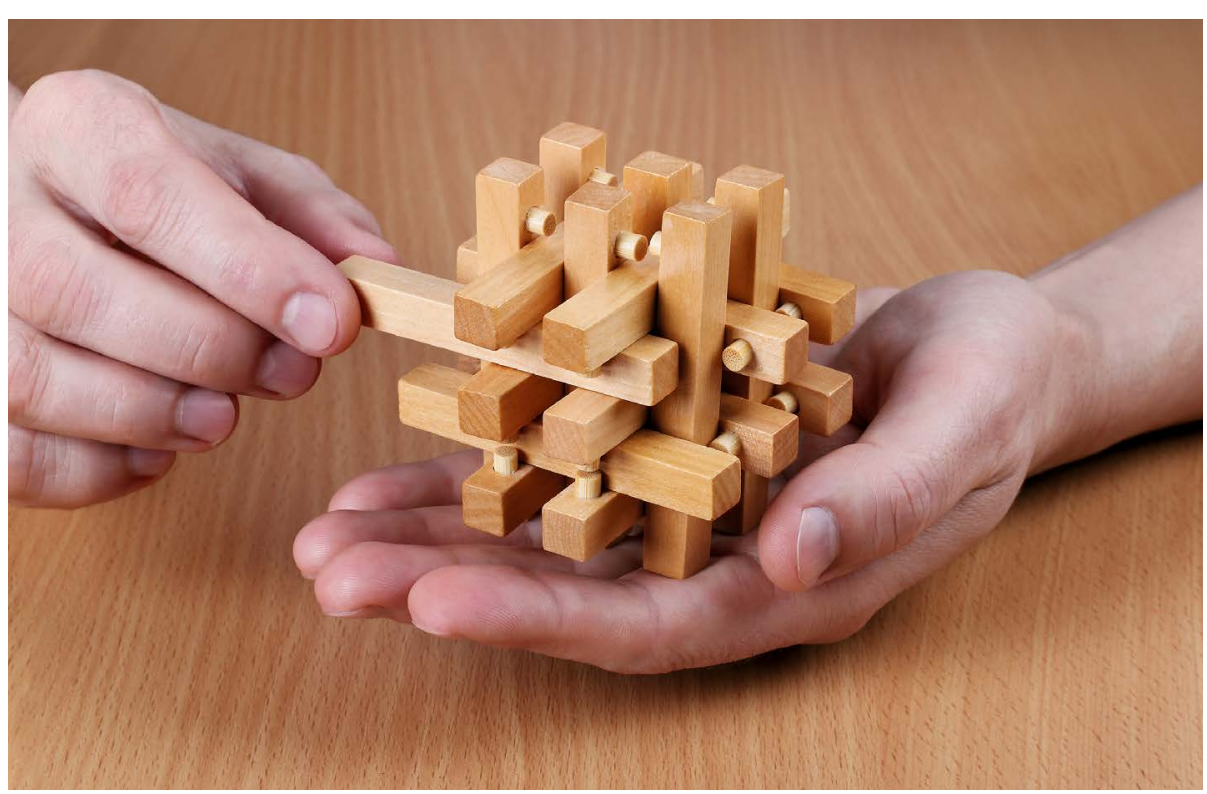

Figure 1 | An interlocking mechanical puzzle. Certain many-body phases of matter, such as Laughlin states, depend on the precise position of all the particles in the system, just like the pieces in an interlocking puzzle. time and length scales. On page 41 , Clark et al. ${ }^{2}$ report the creation of a non-electronic Laughlin state made of composite matter-light particles called polaritons, which are easier to track and manipulate than are electrons.

To picture a Laughlin state, consider a Hall bar, in which such states are usually observed (Fig. 2a). In these devices, electrons that are free to move in a two-dimensional plane are subjected to a strong magnetic field perpendicular to the plane. In classical physics, an electron at any position will start moving along a circular trajectory known as a cyclotron orbit, the radius of which depends on the particle's kinetic 


\section{a Energy-degenerate electronic states}
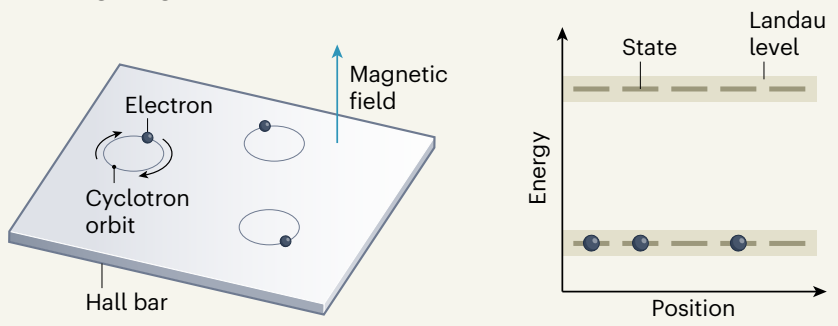

b Energy-degenerate photonic states

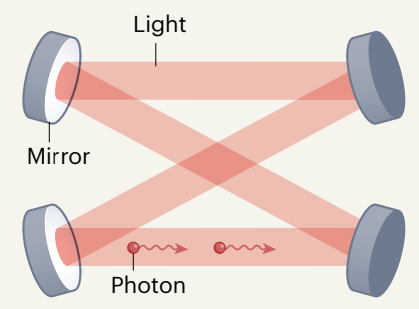

Interactions between electrons
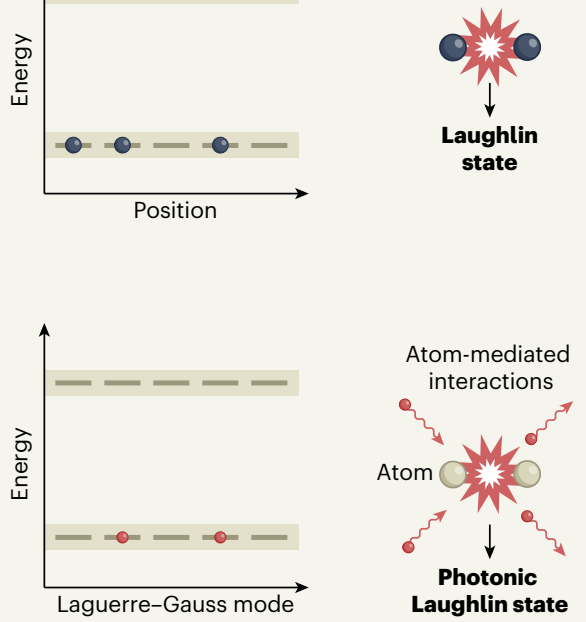

Figure 2 | How to make Laughlin matter. a, An electronic Laughlin state is usually produced in a device called a Hall bar. There, electrons are subjected to a strong perpendicular magnetic field and move along circular trajectories known as cyclotron orbits. This set-up leads to large sets of equal-energy (energydegenerate) electronic states, collectively called Landau levels. When repulsive interactions are added, the electrons enter a Laughlin state. b, Clark et al. ${ }^{2}$ created a photonic Laughlin state by passing light between a system of four mirrors. This arrangement caused modes of light known as Laguerre-Gauss modes to have the same energy as each other, forming analogues of Landau levels. Finally, the authors added atom-mediated interactions between the photons.

energy. In quantum mechanics, the electron's position will still be free, but its orbital radius - and, therefore, its kinetic energy - can be increased or decreased only in discrete steps. This feature leads to large sets of equal-energy (energy-degenerate) states called Landau levels. Non-interacting electrons added to the lowest-energy Landau level can be distributed between the level's energy-degenerate states in many different ways.

Adding repulsive interactions between the electrons constrains the particles' distribution over the states of the lowest Landau level, favouring configurations in which any two electrons have zero probability of being at the same spot. The states described by Laughlin have exactly this property and explain the main features of the fractional quantum Hall effect, whereby electrons in a strong magnetic field act together to behave like particles that have fractional electric charge. This work earned Laughlin a share of the 1998 Nobel Prize in Physics. Laughlin states are truly many-body states that cannot be described by typical approximations, such as the meanfield approximation. Instead, the state of each particle depends on the precise state of all the others, just as in an interlocking puzzle.

To obtain a Laughlin state composed of polaritons, Clark and co-workers needed to add two elements to a photonic system. The first was an equivalent of the magnetic field that would cause photonic states to become energy degenerate. The second was a way for photons to interact with one other, which would force the system to form a Laughlin state, rather than any other combination of degenerate lowest-energy states. In a pioneering set of experiments, the authors' research group had previously demonstrated each element independently ${ }^{3,4}$. In the current work, these elements were combined.

Clark and colleagues used a photonic system called an optical cavity that consisted of four mirrors (Fig. 2b). This set-up caused different

\section{“This work is a milestone for the field of quantum simulation."}

Laguerre-Gauss modes (modes of light that have doughnut-shaped intensity profiles) to have the same energy as each other, forming analogues of Landau levels. Light propagating in the cavity interacted with a cloud of rubidium atoms to promote these atoms to highly excited (Rydberg) states, which are relatively large and interact strongly with one another. The interacting cavity photons and atoms formed the polaritons that the researchers investigated. These polaritons inherited both the energy degeneracy of their photonic part and the interaction of their atomic part, and could, therefore, enter a Laughlin state.

The authors observed the smallest possible Laughlin state - one made of two polaritons.
They demonstrated the properties of this quantum state by carefully studying the light emitted from the cavity. Because the timescales in this system are much longer than those in electronic systems, Clark et al. could measure the number of photons in each light mode, as well as the photons' temporal and spatial correlations, proving the formation of this long-sought state.

This work is a milestone for the field of quantum simulation ${ }^{5,6}$. Physicists in this field build experiments to simulate phenomena occurring in systems that are difficult to manipulate and impossible to model computationally. The simulation system is completely different from that of the original phenomenon, but has the same number of control parameters, and can be used to try to reproduce and understand the underlying principles of the phenomenon.

Clark and colleagues' results further validate the quantum-simulation approach to studying strongly correlated systems. This approach is key to finding materials that have exotic properties and excitations that could revolutionize electronics or quantum computing. The work was implemented on a platform pioneered by the authors' research group, clearing hurdles at which others had stumbled. For instance, the experiment subtly managed to enforce energy degeneracy, which is difficult to attain when the system is not intrinsically symmetric ${ }^{7}$, as was the case here.

The main achievement of this work was to observe the smallest possible Laughlin state made of polaritons, and to carefully characterize it. Future studies could produce larger states that are computationally intractable and probe the excitations of this system. Such advances would test the ability of the platform to accommodate more than two polaritons and to build bigger puzzles of matter and light.

Laura Corman is at X-Rite Europe $\mathrm{GmbH}$, 8150 Regensdorf, Switzerland. e-mail: lauracorman@xrite.com

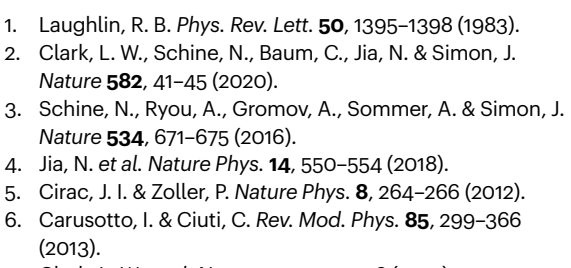

7. Clark, L. W. et al. Nature 571, 532-536 (2019). 